\title{
Advantage of Specimen Heating in FE-EPMA for Performing Quantitative Trace Carbon Analysis in Steel Materials
}

\author{
Yuji Tanaka ${ }^{1}$, Takako Yamashita ${ }^{1}$ and Masayasu Nagoshi ${ }^{2}$ \\ 1. Steel Research Laboratory, JFE Steel Corporation, 1, Kawasaki-cho, Chuo-ku 260-0835, Chiba, Japan \\ 2. Steel Research Laboratory, JFE Steel Corporation, 1-1, Minamiwatarida-cho, Kawasaki-ku, Kawasaki, \\ Kanagawa 210-0855, Japan
}

Controlling the microscopic concentration of trace carbon in steel is critically important since it determines the microstructural development in the phase transformation process. However, precise measurement by electron beam techniques is difficult due to unavoidable hydrocarbon contamination. We developed a custom-made field emission gun electron microprobe analyzer (FE-EPMA) to enable microscopic trace carbon analysis $(\mathrm{C}<1$ mass\%). A multiple $\mathrm{C}-\mathrm{K} \mathrm{X}$-ray signal detection system is used to maximize the weak carbon signal. Conventional and newly-developed anti-contamination devices (ACDs) are installed in parallel to eliminate contamination on the specimen surface. In this study, we evaluate the effect of each ACD on contamination inhibition during EPMA measurements. The effects of a liquid nitrogen $\left(\mathrm{LN}_{2}\right)$ trap, plasma cleaner, and specimen heating as ACDs are investigated.

Commercial pure iron ( $\mathrm{Fe}>99.999$ mass\%) is analyzed in this study. Since this material does not contain carbon, changes in the $\mathrm{C}-\mathrm{K} \mathrm{X}$-ray intensity originate from deposition of contamination on the specimen surface during measurement. The surface was carefully polished with an alumina suspension. An FEEPMA JXA8530F (JEOL) was used to measure the C-K X-ray intensity at an acceleration voltage of 7 $\mathrm{kV}$ and beam current of $50 \mathrm{nA}$. The C-K X-ray signal was collected simultaneously by three identical spectrometers (layered dispersion element LDE6H, JEOL). A plasma asher GV10x (ibss Group Inc., USA) installed in the measurement chamber as an ACD was used to clean the specimen surface and the microscope chamber before a measurement. In addition to the $\mathrm{LN}_{2}$ trap and plasma cleaner, we also investigated the effect of specimen heating for contamination inhibition. The specimen was set in a custom heating holder, and the specimen temperature was kept at approximately $373 \mathrm{~K}$ during the measurement when using the heating holder. Point, line, and map analyses of carbon for the pure iron specimen were performed using various combinations of the above-mentioned ACDs.

Figure 1 shows the carbon elemental mapping for pure iron with the ACDs. The dwell time was set to 50 $\mathrm{ms} /$ pixel and the pixel size was $78 \mathrm{~nm}$. Although the $\mathrm{LN}_{2}$ trap is a widely-used ACD for EPMA[1], SEM, and TEM, its effect is imperfect. The plasma cleaner suppresses contamination deposition, but the X-ray intensity at both edges of the scan direction becomes larger than that at the center of the scanned area due to accumulation of contamination. Specimen heating shows a significant contamination inhibition effect $[2,3]$. The profile of X-ray intensity is constant over the whole map area and provides a reasonable profile for pure iron without an artifact of contamination. Similar results were obtained in the point and line analyses. Figure 2 shows an iterative mapping of carbon on the identical area with all the ACDs. Although the specimen heating technique forms contamination after measurement and cannot remove a pre-existing contamination layer, the results show good repeatability when specimen heating is applied as an ACD. We conclude that a single measurement with a short dwell time with the specimen heating technique is preferable for trace carbon analysis. Under this analytical condition, the effect of contamination on the $\mathrm{C}-\mathrm{K}$ X-ray intensity is negligibly small and quantitative trace carbon analysis for practical materials is possible[4]. 


\section{References:}

[1] B. Buse et al., Microsc. Microanal. 21 (2015), p.594.

[2] T. Yamashita et al., Scientific Reports 6 (2016), 29825.

[3] Y. Tanaka et al., Microscopy doi; http://dx.doi.org/10.1093/jmicro/dfw102

[4] This work is the result of the project of the Innovative Structural Materials Association (ISMA) and was supported by the New Energy and Industrial Technology Development Organization (NEDO).
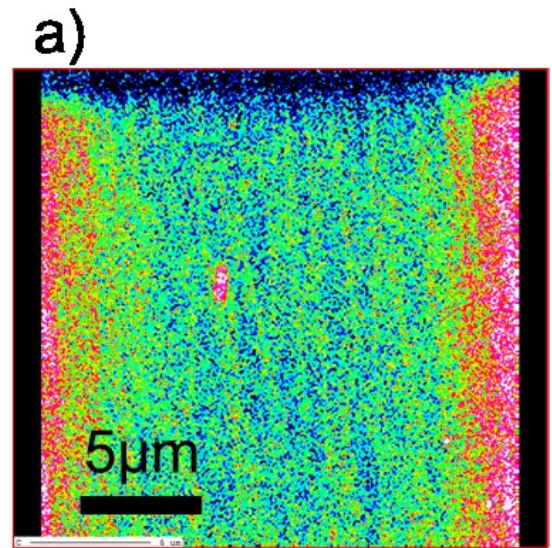
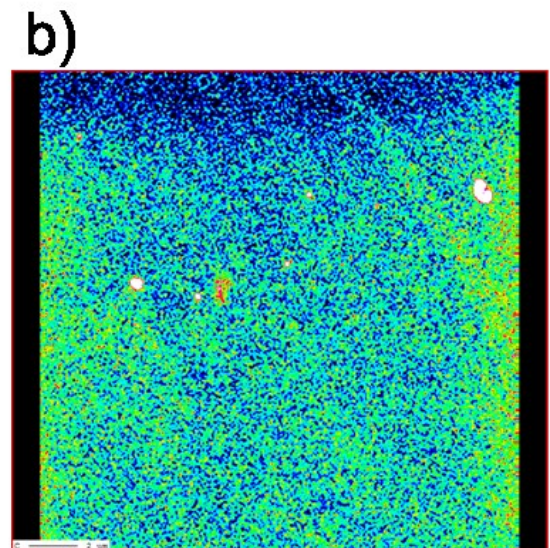

c)

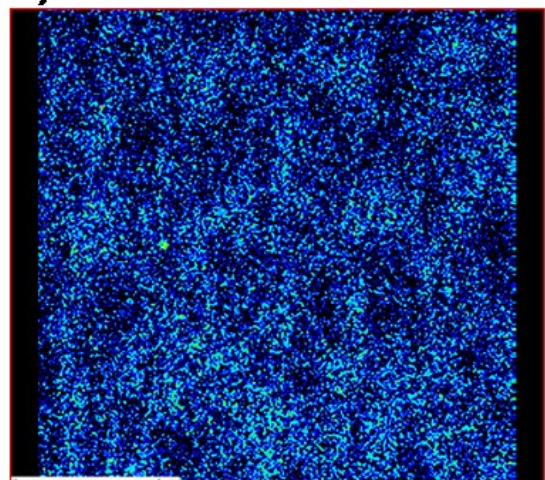

Figure 1. Carbon elemental map for pure iron. a) with the $\mathrm{LN}_{2}$ trap, b) with the $\mathrm{LN}_{2}$ trap and plasma cleaner, and c) with the $\mathrm{LN}_{2}$ trap and specimen heating.
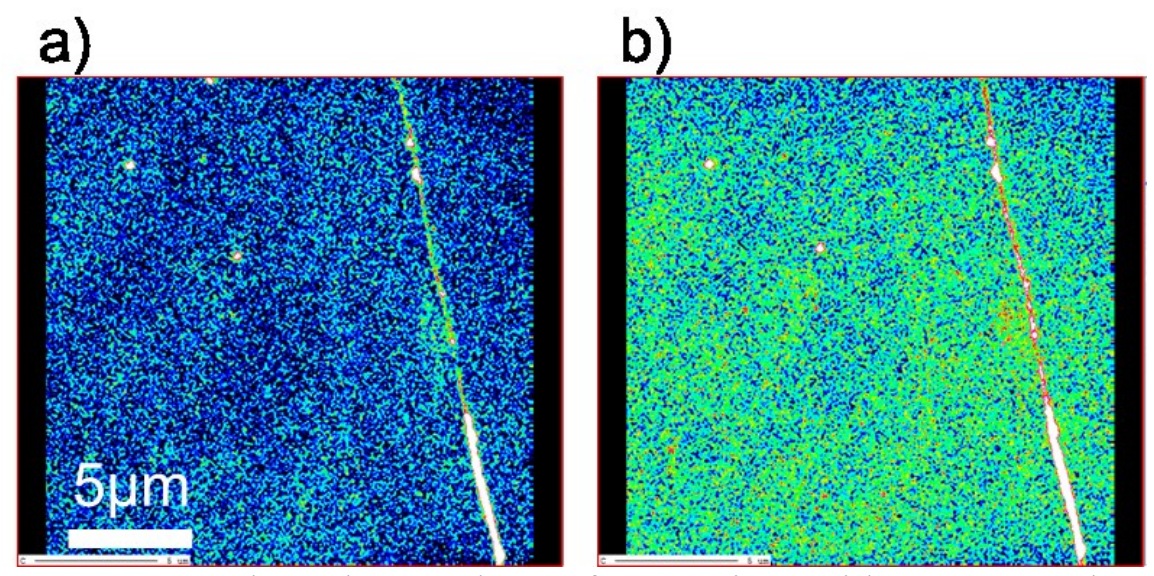

Figure 2. Carbon elemental map for pure iron with $\mathrm{LN}_{2}$ trap, plasma cleaner, and specimen heating as ACDs. a) single measurement. b) the second measurement for identical area of a). 